\title{
Changes in Expression and Distribution of Aplysia Cell Adhesion Molecules Can Influence Synapse Formation and Elimination in vitro
}

\author{
Hui Zhu, Fang Wu, and Samuel Schacher \\ Center for Neurobiology and Behavior, Columbia University College of Physicians and Surgeons, and New York \\ State Psychiatric Institute, New York, New York 10032
}

5-HT or FMRFamide evoke long-lasting changes in the efficacy of sensorimotor (SN-L7) synapses of Aplysia, structural alterations of the presynaptic sensory cell, and cellspecific downregulation in the distribution of the adhesion molecule apCAM. We examined how the cell-specific changes in apCAM might contribute to the formation of new presynaptic varicosities by 5 -HT or the elimination of existing presynaptic varicosities by FMRFamide. We report that the formation of new sensory varicosities is directed by the presence of preexisting zones on the motor axon that are enriched for apCAM. Moreover, there was a further enrichment of apCAM levels at existing sensory varicosities contacting the motor axon beginning at $1 \mathrm{hr}$ and lasting $24 \mathrm{hr}$ after treatment with 5-HT. As was found for synapse formation during the early stages of cell-cell interaction, incubation with anti-apCAM mAb blocked the 5-HTinduced long-term changes in synaptic efficacy and the accompanying changes in sensory neuron structure. Longterm synaptic depression with FMRFamide was accompanied by an overall decline of apCAM levels. Treatment with FMRFamide evoked an even greater decline in apCAM levels at sites of sensory varicosities that preceded the structural changes and persisted especially at sites where sensory varicosities are eliminated. These results suggest that neurotransmitters evoke both cell- and site-specific changes in the levels of adhesion molecules that can influence either the formation or the elimination of presynaptic varicosities that accompany long-term heterosynaptic modulation of a behaviorally relevant synaptic connection.

[Key words: long-term plasticity, synapse formation, synapse elimination, serotonin, FMRFamide, Aplysia cell adhesion molecule, sensory neurons, growth cones, varicosities]

The nervous system of adult animals retains the capacity to undergo structural changes in response to a variety of environmental conditions such as injury or activity-dependent modula-

\footnotetext{
Received Oct. 19, 1994; revised Dec. 22, 1994; accepted Feb. 2, 1995.

We thank Drs. D. Goldberg, J. Koester, I.' Kupfermann, and Z. Sun for their comments on earlier drafts of the manuscript, and Robert Woolley for help in preparing the figures. This work was supported by NIH Grants NS27541 and GM32099 and NSF Grant BNS 9021612

Correspondence should be addressed to Samuel Schacher, Center for Neurobiology and Behavior, Columbia University College of Physicians and Suryeons, New York State Psychiatric Institute, 722 West 168th Street, New York, NY 10032.

Copyright $\odot 1995$ Society for Neuroscience $0270-6474 / 95 / 154173-11 \$ 05.00 / 0$
}

tion of behavior. These latter changes include use-dependent alterations in axon projections and connections in the neocortex of different sensory modalities (Gilbert, 1993; Merzenich and Sameshima, 1993), turnover in the neuronal cell populations and interconnections controlling song learning in birds (AlvarezBuylla et al., 1990; Nottebohm, 1991), and activity-dependent modulation of neuronal structure associated with learning and memory (Bailey and Kandel, 1993). To accomplish these changes in the adult, some of the mechanisms critical during the development of the nervous system that influence axon growth, synapse formation, and stabilization and/or synapse elimination, may be reutilized within the mature nervous system.

One family of cell surface molecules that may play an important role in neuronal plasticity in the mature animal is a member of the immunoglobulin-like family of cell adhesion molecule, neural cell adhesion molecule (NCAM). Specific isoforms of NCAM or NCAM-like surface molecules continue to be expressed in the mature nervous system of both vertebrates (Edelman, 1983; Rutishauser et al., 1988; Edelman and Cunningham, 1991; Seki and Arai, 1993) and invertebrates (Hortsch and Goodman, 1991; Mayford et al., 1992). NCAM isoforms that are prominently expressed during developmental stages associated with axonogenesis and synapse formation (Pollerberg et al., 1985; Chow, 1990; Persohn et al., 1990) are upregulated in the mature system following injury or in the continuously regenerating olfactory system (Daniloff et al., 1986; Miragall et al., 1988; Rutishauser et al., 1988; Key and Akeson, 1991). Recent results on consequences of deleting NCAM or specific isoforms of NCAM in transgenic mice suggest that these molecules may play an important role in the development of specific brain regions, and the normal expression of spatial learning (Tomasiewicz et al., 1993; Cremer et al., 1994). The regulation of the expression and distribution of these molecules in the adult may therefore influence specific cellular changes associated with regeneration and behavioral plasticity (Mayford et al., 1992; Scholey et al., 1993).

In Aplysia, modulation of the expression of NCAM-like cell surface adhesion molecules may contribute to the structural changes in the presynaptic sensory neurons (SNs) and the alterations in synaptic efficacy that accompany long-term changes in defensive withdrawal behaviors (Castellucci et al., 1978; Bailey and Chen, 1983, 1988; Frost et al., 1985). Treatment with 5-HT, a transmitter critical for the expression of behavioral sensitization (Glanzman et al., 1989b) evokes short- and long-term facilitation of SN synapses both in vivo and in vitro (Brunelli et al., 1976; Montarolo et al., 1986; Rayport and Schacher, 1986; 
Dale et al., 1988; Schacher et al., 1990; Pieroni and Byrne, 1992; Clark and Kandel, 1993; Emptage and Carew, 1993). The longterm changes in synapse efficacy are accompanied by structural changes in the neuritic arbor of the presynaptic sensory cells (Glanzman et al., 1990; Schacher et al., 1991) that parallel those observed in vivo with long-term behavioral sensitization (Bailey and Chen, 1983, 1988). Similar treatment of SNs alone with 5-HT transicntly downrcgulates synthesis of all isoforms of apCAM (Barzilai et al., 1989; Mayford et al., 1992), and downregulates the expression of preexisting apCAM on the surface of the SNs (Mayford et al., 1992) via a rapid increase in endocytosis (Bailey et al., 1992a). This selective decrease in apCAM expression and distribution on the surface of the SNs evokes a defasciculation of SN neurites (Peter et al., 1994), which, in turn, can facilitate interaction between neurites of $\mathrm{SNs}$ with neighboring motor neurites leading to the formation of new synaptic connections with the motor target (Zhu et al., 1994). On the other hand, the neuropeptide Phe-Met-Arg-Phe-amide (FMRFamide) evokes short- and long-term depression of sensorimotor connections (Abrams et al., 1984; Belardetti et al., 1987; Mackey et al., 1987; Montarolo et al., 1988; Pieoroni and Byrne, 1992; Small et al., 1992). The long-term change in synaptic function is accompanied by the loss of presynaptic SN varicosities and neurites (Schacher and Montarolo, 1991; Bailey et al., 1992b; Wu and Schacher, 1994). FMRFamide also evokes a rapid downregulation of apCAM expression on the surface of the target motor cell L7 via an increase in endocytosis (Peter et al., 1994) and may contribute to the structural changes in the SNs (Wu and Schacher, 1994).

If both pre- and postsynaptic cells contribute to apCAM expression and to apCAM-ligand molecular interactions at sites of $\mathrm{SN}$-motor cell contact, how do the rapid cell-specific changes in the distribution of apCAM contribute to opposite forms of long-term structural plasticity? One possibility is that each neuromodulator also evokes a decline in apCAM levels at different sites of interaction between the SNs and motor cell L7 and, consequently, contributes either to the formation of new sites of synaptic contact and the stabilization of existing synaptic contacts with 5-HT, or to the destabilization and elimination of existing contacts with FMRFamide. We report here that new SN varicosities, structures known to have the properties of transmitter release sites (Glanzman et al., 1989a, 1990; Schacher et al., 1991), form at sites along the major motor cell axons that are enriched for apCAM both during the early stages of SN-L7 interaction and following 5-HT treatment of mature cultures. Incubation with mAbs against apCAM interferes with the functional and structural changes evoked by 5-IIT. Despite the overall decline in apCAM expressed by the cells in coculture over time, the relatively high level of apC.AM expression at sites of preexisting varicosities is increased further following treatment with 5-HT. By contrast, treatment with FMRFamide results in a significant overall decline of apCAM that is even more pronounced at sites of SN varicosities. This pronounced decline precedes the loss of varicosities. The formation and stability of SN varicosities at sites where apCAM levels on the target cell are relatively high supports the hypothesis that modulating apCAM levels at those sites by neurotransmitters will affect synaptic interactions. The formation of new $\mathrm{SN}$ varicosities is facilitated by 5-HT by altering the relative levels of expression of apCAM in favor of the motor cell at extrasynaptic sites. On the other hand, a significant decrease in the level of apCAM expression on the motor cell at sites of $\mathrm{SN}$ varicosities by
FMRFamide can contribute to the destabilization and elimination of the presynaptic $\mathrm{SN}$ varicosities.

\section{Materials and Methods}

Cell culture. Mechanosensory neurons of Aplysia were isolated from the pleural ganglion dissected from adult animals (70-100 gm) and cocultured with identified gill motor neuron $\mathrm{L} 7$ isolated from the abdominal ganglion of juvenile animals ( $1-3 \mathrm{gm}$; Aplysia mariculture facility at the University of Florida, Miami) as described previously (Rayport and Schacher, 1986). Individual cells were isolated with a segment (100-600 $\mu \mathrm{m}$ ) of their axons intact (Schacher and Proshansky, 1983; Schacher, 1985). Each L7 was cocultured with one sensory cell, and the relative position of each cell was manipulated so that the axon stump of the sensory cell was near $(0-50 \mu \mathrm{m})$ the proximal portion of the L7 axon. The cultures were maintained for either $2 \mathrm{~d}$ (early varicosity formation) or $6 \mathrm{~d}$ (neurotransmitter modulation of function and apCAM levels) in culture medium and under conditions described previously (Proshansky and Schacher, 1983; Rayport and Schacher, 1986; Montarolo et al., 1986; Glanzman et al., 1990; 7hu et al., 1994). Cultures were maintained at $18^{\circ} \mathrm{C}$.

Electrophysiology and application of neuromodulators. The stimulation and recording techniques and the perfusion medium have been described (Dale et al., 1988; Montarolo et al., 1988). Each L7 was impaled intracellularly with a microelectrode $(15-20 \mathrm{~m} \Omega$ ) containing $2.0 \mathrm{M} \mathrm{KCl}$ and held at a potential of $-30 \mathrm{mV}$ below the resting level $(-45$ to $-60 \mathrm{mV})$ to permit accurate measurement of the amplitude of the EPSP. Synaptic potentials were evoked in L7 by stimulating each sensory cell with a brief (0.4-0.6 msec) depolarizing pulse using an extracellular electrode filled with perfusion medium [1:1 by volume of modified L15 (Sigma) and artificial sea water (Instant Ocean), pH = 7.6] and placed near the cell body of the sensory neuron. Cultures were rinsed with perfusion medium $(0.5 \mathrm{ml} / \mathrm{min})$ during recordings and dye injections.

To examine the long-term changes induced by neuromodulators, 5-HT (Sigma) or FMRFamide (Peninsula) were applied on day 5, immediately after the initial recording of the EPSP amplitudes and imaging of the sensory neurites. Neurotransmitters were dissolved in perfusion medium and applied to the cell cultures five times for 5 min (4 $\mu \mathrm{M}$ final concentration), and each application was followed by a 25 min washout. Controls were given five applications of perfusion medium. Following the last washout, cultures were rinsed with culture medium and returned to the incubator. The cultures were reexamined 1 or $24 \mathrm{hr}$ later.

We also examined the expression of long-term changes following 5-HT treatment when cultures were treated with anti-apCAM mAb. Immediately after washout of the last control or 5-HT application, mAb 4F8 (Keller and Schacher, 1990) or IgG2a (Sigma) was applied (250 $\mu \mathrm{g} / \mathrm{ml}$ in perfusion medium) for $3 \mathrm{hr}$. Cell cultures were washed $2 \times$ with perfusion medium, rinsed with culture medium, and returned to the $18^{\circ} \mathrm{C}$ incubator for $24 \mathrm{hr}$ until reexamination.

ApCAM immunofluorescence, dye injection, and cell imaging. SNL7 cultures ( $1 \mathrm{~d}$ or $5 \mathrm{~d}$ ) were rinsed $2 \times$ at 30 min intervals with perfusion mediun and exposed for 30 mini to FITC-conjugated anti-ApCAM monoclonal antibody $(4 \mathrm{E} 8,20 \mu \mathrm{g} / \mathrm{ml})$ dissolved in perfusion medium (Keller and Schacher, 1990; Mayford et al., 1992; Peter et al. 1994; Wu and Schacher, 1994; Zhu et al., 1994) to examine apCAM expression and distribution along the original proximal axons of $\mathrm{L7}$ where most SN varicosities are located. In addition, because the original motor axon is a static nongrowing structure, apCAM staining at precise locations of SN-L7 interactions (such as SN varicosities) can be monitored over time. Unbound $\mathrm{mAb}$ was washed out with perfusion medium. Due to the relatively low turnover rate of apCAM, the fluorescent signal intensity $1 \mathrm{~d}$ later remains strong.

After recording the amplitude of EPSP on day 1 and day 2 (early interaction) or day 5 and 6 (neuromodulator-induced long-term changes), lissamine-rhodamine B (5\% in water; High Wycombe, England) was iontophoretically injected into sensor y cells with $0.4-0.8 \mathrm{nA}$ hyperpolarizing current pulses $(500 \mathrm{msec}$ at $1 \mathrm{~Hz})$ until the SN cell body turned light red (Glanzman et al., 1991; Bank and Schacher, 1992; Zhu et al., 1994). To examine the early changes in structure and distribution of apCAM, SNs were reinjected with rhodamine $1-2 \mathrm{hr}$ after the last application of neurotransmitter. In cultures treated with $4 \mathrm{E} 8$ or IgG after control or 5-HT treatments, SNs were injected with fluorescent dye 5(6)-carboxyfluorescein (Molecular Probes, $6 \%$ in $0.44 \mathrm{M} \mathrm{KOH}, \mathrm{pH}$ 
$=7.0$ ) on days 5 and 6 using hyperpolarizing current pulses of $0.3-0.5$ $\mathrm{nA}, 500 \mathrm{msec}$ at $1 \mathrm{~Hz}$ for $5-6 \mathrm{~min}$.

For most experiments (except examining the effect of $4 \mathrm{E} 8$ on longterm facilitation by $5-\mathrm{HT}$ ), two sets of fluorescent images (rhodamine fluorescence images of SN structures and FITC staining revealing level of expression and distribution of apCAM) and Nomarski contrast images of the same view areas were examined. Images were taken of areas along the original major axons of L7 on either day 1 and day 2 or on day 5 (twice for early changes associated with transmitter treatments that evoke the long-term changes) and on day 6 (for long-term changes) using a Nikon Diaphot microscope with a Dage SIT camera. Images were processed by a Dell 310 computer with a PC Vision Plus frame grabber and subsequently stored on a Panasonic optical disc drive. Alignment of the live view area for both Nomarski contrast views of the I.7-SN neurites and epifluorescent views of the dye-filled SN neurites with previous Nomarski and epifluorescent images was aided by computer, and with fine adjustments made with manual rotation of the culture dish. Each focal plane plotographed with SN processes (rhodamine epifluorescence) was also photographed for apCAM distribution (fluorescein epifluorescence). Illumination used for obtaining fluorescence images was kept as low as possible to prevent photodamage. To minimize differences in imaged SN structures that might arise as a result of differences in the extent of dye filling, light intensities used on the second day were adjusted to match the intensity of the stored images taken on the first day. Light intensity used to take the FITC-4E8 epifluorescence pictures was set at the same level for all experiments to facilitate comparison of fluorescent signals as an index of apCAM expression levels and distribution. Micrographs of the images were made with a Panasonic or Sony printer.

Quantification of ApCAM distribution and change in structure. Previous studies indicated that the most favorable substrate for the growth of sensory neurites with varicosities was along the stationary nongrowing original proximal motor axons (Glanzman et al., 1989, 1990; Zhu et al., 1994). We measured the FITC-4E8 fluorescence intensities for each local area with a sensory varicosity along the major axon of $\mathbf{L} 7$, and compared this value with the average fluorescence intensity for each segment of the L7 axon. To locate sites of SN varicosities along the motor axon, we first specified the area of the entire original motor axon segment on the corresponding Nomarski contrast image (not shown, but see Figs. 4, 5 in Zhu et al., 1994). The small area of each SN varicosity was outlined and fixed using the corresponding rhodamine images of SN Ileurites. The average pixel intensities of the PITC staining for these small areas of varicosities were measured at both time points and compared to the average pixel value of the $\mathrm{L} 7$ axon segment. The images were viewed at a magnification of $2500 \times$. Because the original motor axon can have a diameter of about $25 \mu \mathrm{m}$, it often required several different focal planes to image all of the labeled $\mathrm{SN}$ structures in a given view area. The FITC-4E8 fluorescent images were also taken at exactly each of these focal planes to facilitate accurate comparison of apCAM levels at varicosities to the average intensity of the entire segment of the L7 axon. In addition to using the Nomarski contrast images, areas underlying new varicosities or formerly containing old varicosities were identified using other fixed epifluorescent sensory structures. A varicosity was considered new either on day 2 or day 6 if the structure was not located within a $3 \mu \mathrm{m}$ radius in the same region of the motor axon on either day 1 or day 5. A varicosity was considered to be preexisting or eliminated if the structure was present at both time points or absent at the second viewing within a $3 \mu \mathrm{m}$ radius within the same region of the motor axon. Where 5-HT-induced long-term facilitation was tested in the presence or absence of $4 \mathrm{E} 8$, net changes in varicosity numbers were measured. Structures greater than or equal to $2.0 \mu \mathrm{m}$ that were slightly elongated spheres connected by narrow neuritic necks were counted as varicosities (Bailey and Chen, 1983, 1988). All measurements of FITC-4E8 fluorescence and counting of varicosities were performed by an individual who was unaware of treatment or EPSP amplitudes.

Analysis of data. All data in the text and figures are presented as mean \pm SEM. Stude t's $t$ tests (two tailed) or ANOVAs followed by corrected multicompar on tests (Dunnett's) were used to measure the significance of the diffe. nces observed with treatments in the changes of EPSP, of the varicosit numbers, and the significance of changes in overall apCAM levels or a CAM levels at varicosities.

\section{Results}

$S N$ varicosities form at ApCAM "hot spots" on $L 7$

Mechanosensory cells of Aplysia rapidly and reliably form chemical connections with the gill and siphon motor cell L7 in cell culture. EPSPs in L7 are evoked by stimulation of the SN by $12 \mathrm{hr}$ in culture, and increase in amplitude for $4 \mathrm{~d}$ (Glanzman et al., 1990; Bank and Schacher, 1992; Zhu et al., 1994). During the reestablishment of sensorimotor connections, SN neurites prefer to extend and form varicosities with transmitter release sites on the original proximal segments of the motor axon (Glanzman et al., 1989b; Zhu et al., 1994) where apCAM is expressed at high levels compared to distal motor neurites or SN neurites (Wu and Schacher, 1994; Zhu et al., 1994). Since the level of apCAM expression is high at established sites of synaptic interaction both in vivo and in vitro (Keller and Schacher, 1990), we used a double-label fluorescence method (Keller and Schacher, 1990; Zhu et al., 1994) to first examine whether new SN varicosities form at random sites along the proximal motor axons during the early stages of sensorimotor interaction (day 1 and day 2 in culture) or at specific sites along the motor axon that already contain high levels of apCAM. We then examined whether new $\mathrm{SN}$ varicosities that form in association with long-term facilitation of stable sensorimotor connections following treatment with 5-HT on day 5 in culture (Glanzman et al., 1990) form at sites on the proximal motor axon that also contain high levels of apCAM.

Both $1 \mathrm{~d}$ and $5 \mathrm{~d}$ SN-L7 cultures were first stained with FITC-anti-apCAM mAb (4E8; Keller and Schacher, 1990) before recording the initial amplitude of the EPSPs evoked by stimulation of the sensory cell with an extracellular electrode (see Materials and Methods). The binding of the low level of FITC-labeled $\mathrm{mAB}$ had no effect on the pattern of growth during early period of synapse formation, on the functional and structural changes evoked by 5-HT in mature cultures, or on the amplitude of the EPSP in control cultures (see below). Each SN was stained by intracellular injection of lissamine-rhodamine (Keller and Schacher, 1990; Glanzman et al., 1991; Bank and Schacher, 1992; Zhu et al., 1994) before imaging both the SN neurites and varicosities in contact with the motor axon, and the intensity of anti-apCAM mAb staining along the same regions of the motor axon.

Ncw SN varicosities form primarily at local preexisting areas of high apCAM staining on the surface of L7 (Fig. 1). SN growth cones extend along the surface of the proximal motor axons and formed varicosities at sites on the motor axon that stain on the average with significantly higher intensity $(140.8 \pm$ $4.2 \% ; N=6$ cultures and 52 new varicosities; $p<0.001$ ) than the average overall intensity of anti-apCAM staining along the proximal motor axon (Fig. 1A2-A4). Even new varicosities established at sites with low preexisting levels of apCAM form at sites that are stained at a relatively higher intensity than the immediately surrounding areas of the motor axon (see Fig. 1A3). As previously reported for established varicosities, the level of apCAM staining on day 2 was enriched significantly for all varicosities $(128.5 \pm 3.3 \% ; p<0.005)$ compared to the overall level of staining along the motor axon (Fig. 1A3,A4).

New sensory varicosities that form by day 6 following treatment with 5-HT that evokes long-term facilitation of the connection also formed at sites along the proximal motor axon that had higher levels of apCAM staining compared to surrounding areas of the motor axon (Fig. 1B). These sites had $137.3 \pm$ 
Figure 1. SN varicosities form at sites enriched for apCAM on the motor cell axon. $A$. New varicosities form initially at sites with high levels of apCAM. $A I$ and $A 3$, Rhodamine fluorescent views of $\mathrm{SN}$ neurites, varicosities, and growth cones (especially in $A 1$ ) regenerated by day 1 and day 2 , respectively, on the surface of a segment of a L7 axon (not shown). Arrows in $A 3$ point to some of the new sensory varicosities. $A 2$ and $A 4$, Pseudocolor representation (red is high intensity and blue is low intensity) of the FITC-antiapCAM staining (an index of apCAM distribution) in the same area. The $a r$ rows in $A 4$ point to the sites of the new $\mathrm{SN}$ varicosities indicated in $A 3$. Note that some of the newly formed SN structures form at apCAM "hot spots" on L7's axon (arrows in A4), and these "hot spots" existed prior to the formation of the new $\mathrm{SN}$ varicosities (A2). Some of the other varicosities form at sites with relatively higher apCAM expression compared to the immediately surrounding area of the motor axon. $B$, Long-term facilitation by $5-\mathrm{HT}$ is accompanied by formation of $\mathrm{SN}$ varicosities at local apCAM "hot spots" on the motor axon. $B I$ and $B 3$, Rhodamine fluorescent views of $\mathrm{SN}$ neurites and varicosities contacting motor axon before (day 5 in culture; $B 1$ ) and $24 \mathrm{hr}$ after (B3) treatment with 5-HT. Arrows in $B 3$ point to new varicosities. $B 2$ and $B 4$, Pseudocolor representation of FITC-anti-apCAM staining of the same area before $(B 2)$ and after $(B 4)$ treatment with 5-HT. Note that some of the new SN varicosities (arrows in B3) form at sites enriched for apCAM along L7 (arrows in B4). Note that the new varicosities at right arrow form at a site with relatively low apCAM levels, but is higher compared to the adjacent areas along the L7 axon. Scale bar, $30 \mu \mathrm{m}$.
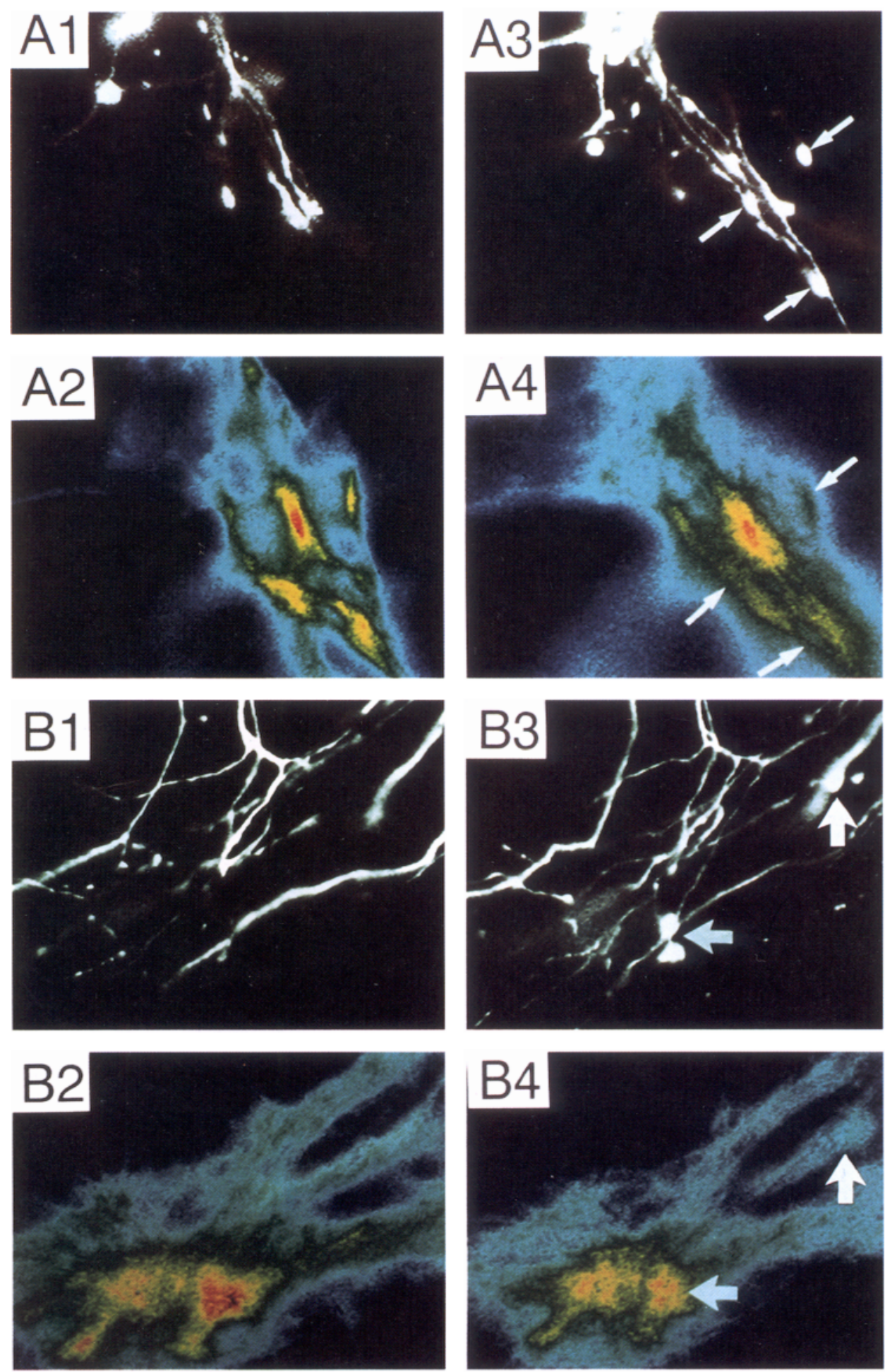

2.3\% ( $N=7$ cultures) higher intensity fluorescence staining compared to the overall intensity along the motor axon $(p<$ $0.001)$. As with new varicosities during the initial interactions, some varicosities form at sites that show higher intensity of staining relative to the neighboring area of the motor axon (right arrow in Fig. 1B3,B4). The formation of new presynaptic varicosities either during the initial interaction between the cells or following 5-HT appear to be regulated or directed by local differences in the level of apCAM expression on the postsynaptic target motor cell. We therefore examined whether application of "blocking" levels of anti-apCAM mAb (see below) interferes with the long-term functional and structural changes evoked by 5-HT.

Anti-ApCAM mAb blocks structural and functional changes evoked by 5-HT

High levels of anti-apCAM mAb or their Fab fragments (250 $\mu \mathrm{g} / \mathrm{ml}$ for $3 \mathrm{hr}$ ) reduce cell-cell interactions for SNs or motor neurons. Incubating isolated SNs or motor neurons with antiapCAM mAb or the Fab fragments reduces fasciculation of 
A

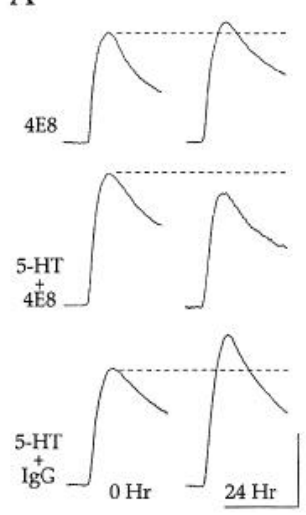

B

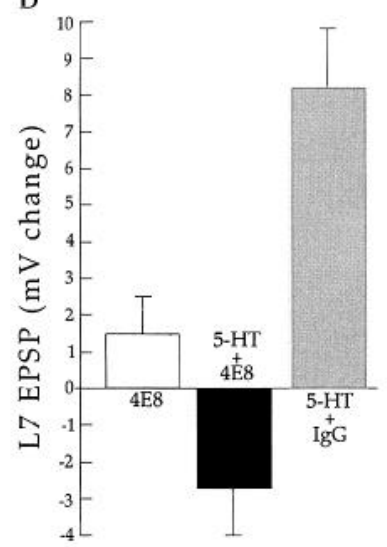

C

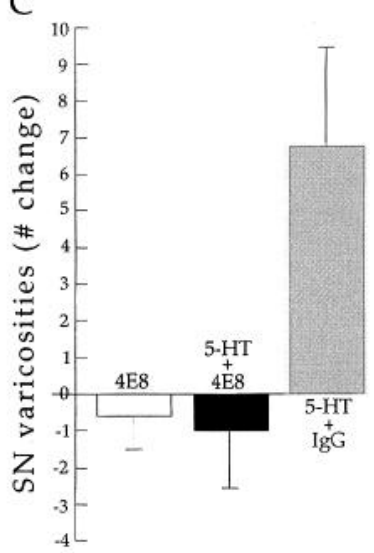

Figure 2. Anti-apCAM mAb blocks long-term changes evoked by 5-HT. A, The long-term increase in the amplitude of the EPSPs in L7 by 5-HT is blocked by mAb 4E8. EPSPs before $(0 \mathrm{hr})$ and after $(24 \mathrm{hr})$ treatment with either anti-apCAM mAb alone (4E8) or $4 \mathrm{E} 8$ after treatment with 5-HT $(5-H T+4 E 8)$ were increased or decreased slightly, while adding control IgG after treatment with 5-HT $(5-H T+I g G)$ had little effect on the typical increase in the EPSP amplitude evoked by 5-HT. The vertical bar equals $15 \mathrm{mV}$ and the horizontal bar equals $15 \mathrm{msec}$. $B$, Summary of the changes in the amplitude of the EPSP for the different groups. The height of each bar represents mean \pm SEM of the change in the EPSP amplitude. An ANOVA indicated a significant effect of treatment $[F(2,15)=21.343, p<0.001]$. Multicomparison $t$ tests (Dunnett's) indicated that the change with 5-HT $+4 \mathrm{E} 8$ was not different than the change with 4E8 alone $(t=1.326, p>0.3)$. EPSP was increased significantly with 5-HT $+\operatorname{IgG}(t=4.118, p<0.01)$. $C$, Summary of net changes in the number of SN varicosities with different treatments. Each bar represents the mean \pm SEM of the net change in the number of SN varicosities. An ANOVA indicated a significant effect of treatment $[F(2,15)=16.013$, $p<0.005$ ]. Multicomparison $t$ tests (Dunnett's) indicated that the change with 4E8 +5 -HT was not significantly different than 4E8 alone, while the increase in varicosities when IgG was applied after 5-HT was significant $(t=3.329, p<0.01)$.

growth cones with homologous neurites (Keller and Schacher, 1990; Mayford et al., 1992; Peter et al., 1994). Preincubation of the motor target alone with anti-apCAM mAb interfered with the normal level of interaction between SN growth cones and the proximal motor axon and reduced significantly the efficacy of new synaptic connections (Zhu et al., 1994). We therefore examined whether similar treatments interfered with the longterm functional and structural changes evoked by 5 -HT. The amplitudes of the EPSPs were recorded and the structure of SN neurites interacting with the proximal motor axon were imaged following intracellular injections with 5-(6)carboxyfluorescein before and $24 \mathrm{hr}$ after control and 5-HT treatments.

Anti-apCAM mAb (4E8) applied for $3 \mathrm{hr}$ after 5-HT treatment blocked long-term changes in the efficacy of the connections (Fig. $2 A, B$ ) and in the formation of new SN varicosities (Fig. $2 C$ ). The same application of anti-apCAM mAb after control treatment had little long-term effect on the EPSP or on the structure of the sensory neurites. Incubation with either anti-apCAM $\mathrm{mAb} 4 \mathrm{E} 8$ or the control antibody (IgG) had no effect on shortterm facilitation evoked by a single brief application of 5-HT, or on other short-term modulations (homosynaptic or heterosynaptic depression) of the connection (data not shown). For cultures treated with mAb 4E8 alone, the amplitude of the EPSPs changed slightly (about $7 \%$ ) by $1.4 \mathrm{mV} \pm 1.1$ ( $N=6$ cultures), and the number of varicosities changed by $-0.6 \pm 0.9$. When $\mathrm{mAb} 4 \mathrm{E} 8$ was added to the cultures for $3 \mathrm{hr}$ after repeated applications of 5-HT, no long-term increases in function or structure were produced; treatment evoked a change of $-2.7 \mathrm{mV} \pm$ 1.2 (about $-13 \%$ ) in the amplitude of the EPSP, and a net change of $-1.1 \pm 1.6$ varicosities $(N=6$ cultures $)$. By contrast, adding control IgG after treatment with 5-HT had little effect on the long-term changes normally evoked by 5 -HT alone; the amplitude of the EPSP increased by $8.2 \mathrm{mV} \pm 1.7$ (about $40 \%$ ) and the number of $\mathrm{SN}$ varicosities increased by $6.8 \pm 2.7(N$ $=6$ cultures). These results, coupled with earlier findings on the possible role of changes in apCAM levels with synapse forma- tion and with long-term depression (Wu and Schacher, 1994; Zhu et al., 1994), suggest that interfering with the levels of expression of one type of cell surface molecule on the SN neurites and the proximal motor axon can influence the formation of new SN varicosities following treatment with 5-HT.

\section{Differential changes in ApCAM distribution evoked by 5-HT and FMRFamide}

Previous studies suggest that the neuromodulators alter the distribution of apCAM on the surface of either the SN (5-HT) or the motor cell (FMRFamide) as soon as $30 \mathrm{~min}$ after completing the treatments that evoke the respective long-term changes (Bailey et al., 1992; Mayford et al., 1992; Peter et al., 1994; Wu and Schacher, 1994). Since these earlier studies only examined changes in apCAM distribution on isolated SNs or motor cells, we examined how the transmitters affected the expression and distribution of preexisting apCAM on the surfaces of the cells in cocultures as the reconstituted circuits undergo long-term plasticity. We compared the changes in overall apCAM expression following control or experimental treatments, and the changes in relative levels of apCAM expression at sites of preexisting varicosities, new varicosities (see Fig. 1), and those that were eliminated.

As previously reported, the neuromodulators evoked a significant change in the amplitude of the EPSP after $24 \mathrm{hr}$. Treatment with 5-HT ( $N=7$ cultures) evoked a significant increase ( $p<$ $0.01)$ of $42.6 \pm 6.3 \%$ in the amplitude of the EPSP compared to a change of $-1.0 \% \pm 5.6 \%$ ( $N=11$ cultures) for control treatment. By contrast, FMRFamide $(N=7$ cultures) produced a significant decline $(p<0.01)$ of $-34.4 \pm 6.4 \%$ in the EPSP amplitude.

The overall level of apCAM expression was affected by treatment. Since the proximal motor axons contribute most of the apCAM signal (Zhu et al., 1994), and apCAM levels on the motor axon are decreased by FMRFamide (Peter et al., 1994; Wu and Schacher, 1994), it was not surprising that FMRFamide 
Ohr

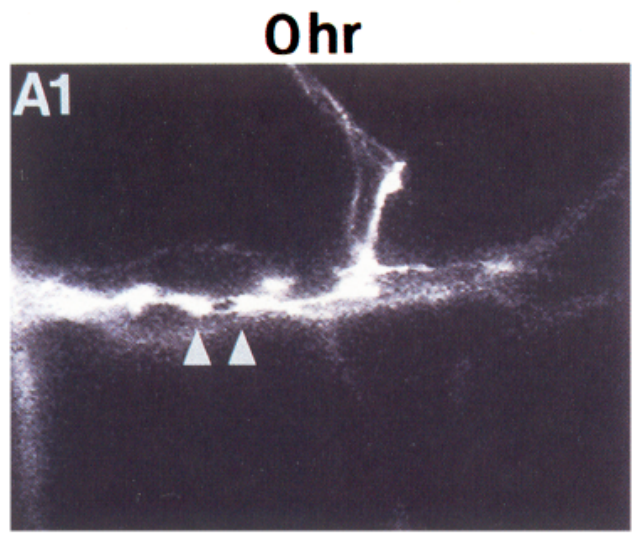

Figure 3. FMRFamide and 5-HT evoke changes in apCAM levels at $\mathrm{SN}$ varicosities. A, FMRFamide evoked a significant decline in apCAM levels at SN varicosities. $A I$ and $A 3$, Rhodamine fluorescent view of SN structures along a segment of motor axon before $(0 \mathrm{hr}$; $A 1)$ and after (24 hr; A3) treatment with FMRFamide. A number of varicosities (four) are lost, and the location of two of them are indicated by the arrowheads. $A 2$ and A4, Pseudocolor representation of anti-apCAM FITC signal of the same region before $(A 2)$ and after (A4) FMRFamide. Note that there is a general decrease in apCAM expression following FMRFamide treatment, and this decrease is particularly significant at sites where SN varicosities were eliminated (open arrows in $A 2$ point to regions with yellow intensity and the same regions in $A 4$ have blue intensity). $B$, 5-HT evoked a significant increase in apCAM levels at preexisting $\mathrm{SN}$ varicosities. $B 1$ and $B 3$, SN structures along a segment of motor axon before $(B 1)$ and after $(B 3)$ treatment with 5-HT. Arrows point to the same $\mathrm{SN}$ varicosities that remain in contact with L7. B2 and B4, Pseudocolor representation of anti-apCAM FITC signal of the same region before (B2) and after (B4) 5-HT. Note that there is very little change in the overall level of apCAM (some regions decrease while others increase) following treatment (compare B2 to B4). ApCAM levels in areas with varicosities have increased (compare areas indicated by the arrows in B2 with dark blue intensity with the same areas in $B 4$ that now have light blue-green intensity). Scale bar, $30 \mu \mathrm{m}$.
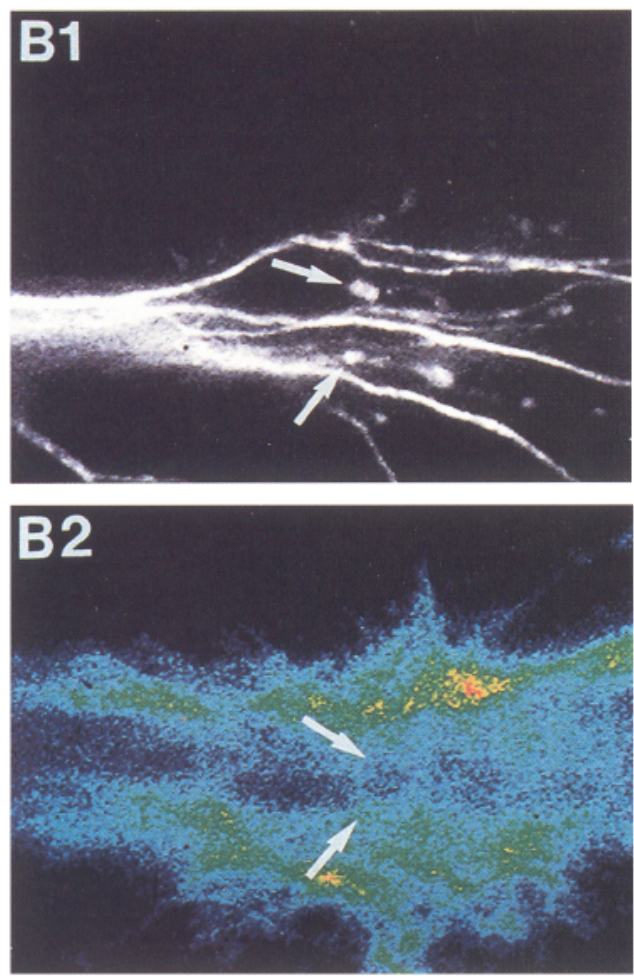
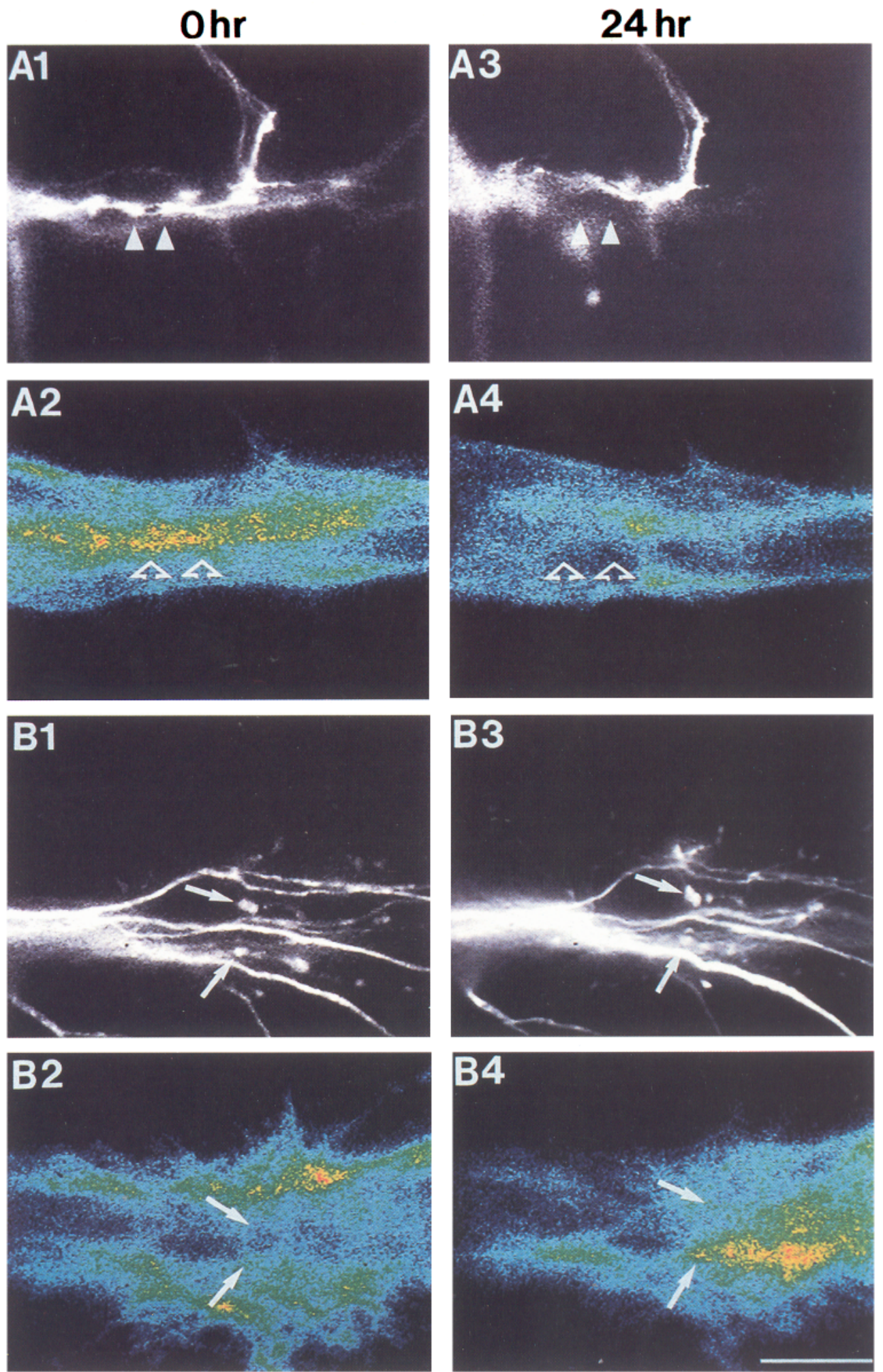

evoked a significant decline in overall fluorescence along the proximal motor axon in SN-L7 cocultures (Figs. 3A, 4A) compared to control or 5-HT treatment (Fig. 4A). Overall intensity of apCAM fluorescence along the proximal motor axon declined by $-16.6 \pm 4.3 \% 24 \mathrm{hr}$ following control treatment and declined by $-19.0 \pm 5.2 \%$ following 5 -HT, compared to a decline of $-35.6 \pm 4.0 \%$ with FMRFamide. The changes in fluores- cence intensity at sites containing $\mathrm{SN}$ varicosities differed for both 5-HT and FMRFamide when compared to controls. Before treatment, anti-apCAM fluorescence intensity was enriched at varicosities for all cultures compared to overall fluorescent signal from the same region of the proximal motor axon; $120.0 \pm$ $3.2 \%$ for controls, $118.9 \pm 3.7 \%$ for $5-\mathrm{HT}$, and $123.0 \pm 4.9 \%$ for FMRFamide (Fig. 4B). One day after control treatment, the 
A

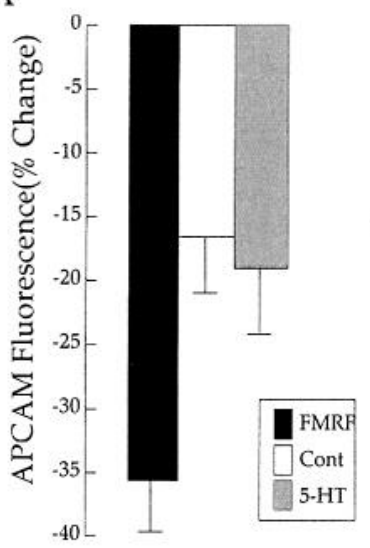

B

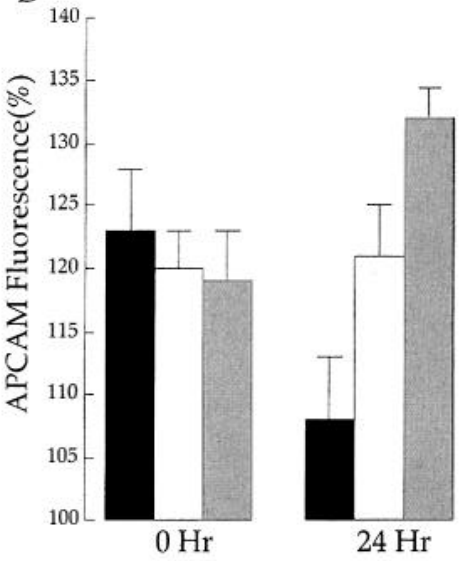

Figure 4. Summary of the changes in apCAM expression $24 \mathrm{hr}$ following treatments. $A$, Overall apCAM levels along the proximal motor axon containing $\mathrm{SN}$ neurites and varicosities is reduced significantly by FMRFamide. The height of each bar represents the mean \pm SEM of the percent change in apCAM fluorescence following different treatments. An ANOVA indicated a significant effect of treatment $[F(2,22)$ $=4.762, p<0.02]$. Multicomparison $t$ tests (Dunnett's) indicated that the change with 5-HT was not different than control while FMRFamide decreased significantly apCAM levels $(t=2.897, p<0.01)$. B. Both 5 -HT and FMRFamide alter apCAM levels at SN varicosities. The height of each bar is the mean \pm SEM in the relative apCAM fluorescence intensity at preexisting SN varicosities before $(0 \mathrm{hr})$ and after $(24$ $h r$ ) treatments. A two-factor ANOVA indicated a significant effect of treatment $[F(2,22)=31.368, p<0.001]$. An ANOVA at each time point indicated that there was no significant difference between the groups on $0 \mathrm{hr}[F(2,22)=0.556, p>0.55]$ while there was a significant effect of treatment at $24 \mathrm{hr}[F(2,22)=5.529, p<0.02]$. Multicomparison $t$ tests (Dunnett's) indicated that the change with FMRFamide and 5-HT were significantly different than control $(t=2.098, p<0.05$ for FMRFamide; $t=2.047, p<0.05$ for 5-HT).

relative level of fluorescence intensity at the same varicosity sites was unchanged, $120.8 \pm 3.8 \%$, indicating that turnover or loss of preexisting apCAM at the varicosities was comparable to the turnover or loss of apCAM for the entire region of the proximal motor axon. In contrast, 5-HT evoked a significant enrichment of anti-apCAM fluorescence signal at the sites of preexisting varicosities relative to the overall fluorescence (Figs. $3 B, 4 B$ ) to $131.6 \pm 2.4 \%$, while treatment with FMRFamide evoked a significant decline in anti-apCAM fluorescence at sites of preexisting varicosities relative to overall fluorescence to $107.9 \pm 5.4 \%$ (Figs. $3 B, 4 A$ ). These changes suggest that 5 -HT decreases turnover of apCAM at preexisting varicosities compared to the overall decline of apCAM on the surface of the cells, while FMRFamide increases the turnover of apCAM levels at sites of preexisting varicosities compared to the overall decline. Sites along the motor axon that had lost $\mathrm{SN}$ varicosities had the largest decline in relative apCAM levels to $99.7 \pm 7.0 \%$ ( 34 varicosities lost in the seven cultures treated with FMRFamide). By contrast, new varicosities formed following 5-HT (45 varicosities in seven cultures) showed a relatively high level of apCAM fluorescence $(137.3 \pm 2.3 \%$; see Fig. $1 B)$.

These site-specific changes in the distribution of apCAM at preexisting varicosities evoked by the different neurotransmitters occur rapidly and precede the changes in structure. A significant decline in overall levels of apCAM was observed by $1 \mathrm{hr}$ after the last application of FMRFamide (Figs. 5A, 6A) compared to control or 5-HT treatment (Fig. 6A). Overall intensity of apCAM fluorescence along the proximal motor axon declined by -9.0

$\pm 2.5 \% 1 \mathrm{hr}$ following control treatment $(3 \mathrm{hr}$ after the onset of the treatment) and declined by $-9.1 \pm 2.2 \%$ following $5-\mathrm{HT}$, compared to a decline of $-20.8 \pm 2.3 \%$ with FMRFamide. The change in apCAM levels at the varicosities $1 \mathrm{hr}$ after treatment paralleled the changes observed at $24 \mathrm{hr}$ after treatment (Figs. $4 B, 6 B)$. One hour after control treatment ( $N=10$ cultures), the relative level of fluorescence intensity at the same varicosity sites was unchanged, $125.2 \pm 1.8 \%$ versus $125.4 \pm 1.6 \%$. Whereas $\mathrm{SN}$ neurites in $\mathrm{SN}$-alone cultures undergo a significant decline in apCAM expression after 5-HT, apCAM levels at old varicosity sites in $\mathrm{SN}-\mathrm{L} 7$ cultures begin to change with a small increase in apCAM staining intensity $1 \mathrm{hr}$ after treatment with 5 -HT $(N=10), 120.0 \pm 3.0 \%$ to $127.5 \pm 3.4 \%$ (Figs. $5 B, 6 B$ ). By contrast, $1 \mathrm{hr}$ after treatment with FMRFamide $(N=10)$ there is a significant decline in apCAM staining, $120.1 \pm 2.2 \%$ to $103.1 \pm 1.6 \%$ (Figs. $5 A, 6 B$ ). Thus, the stability and formation of new varicosities with 5-HT and the lability of preexisting varicosities with FMRFamide are correlated with the site-specific changes in the distribution of apCAM produced by the neurotransmitters.

\section{Discussion}

The results reported here, coupled with previous findings (Bailey et al., 1992; Mayford et al., 1992; Hu et al., 1993; Peter et al., 1994; Wu and Schacher, 1994; Zhu et al., 1994), suggest that local cell- and site-specific changes in the distribution of one class of cell adhesion molecules can contribute to the different forms of structural plasticity accompanying long-term facilitation and depression of a behaviorally relevant synaptic connection in Aplysia. Sites of synaptic interaction between the sensory and motor cells are enriched for apCAM (Keller and Schacher, 1990). Thus, the rapid transmitter-induced changes in local apCAM levels may contribute to the subsequent structural changes. Hot spots of apCAM that are present on the surface of the motor axon prior to reinnervation by the $\mathrm{SN}$ can influence the location and/or formation of presynaptic varicosities that are known to contain transmitter release sites (Glanzman et al., 1989, 1990; Schacher et al., 1991). Blocking apCAM on the motor cell with anti-apCAM antibody prior to the initial interaction between the $\mathrm{SN}$ and the motor cell did not interfere with SN growth per se, but interfered with the normal pattern of SN neurite interaction with the motor axon and reduced significantly the efficacy of the connections (see Fig. 4 in Zhu et al., 1994). Adding anti-apCAM mAb to cultures immediately after treatment with 5-HT also interfered with the formation of new varicosities and the long-term enhancement in the efficacy of the sensorimotor connection, but without affecting short-term synaptic plasticity. These cell adhesion molecules may therefore represent one class of membrane proteins in Aplysia involved with synapse formation and maintenance. The role of apCAM in the reestablishment of $\mathrm{SN}$ varicosities at sites along the proximal motor axon parallels the roles of surface molecules and receptors during the reestablishment of the neuromuscular junction at the old endplate sites (McMahan et al., 1992; Hall and Sanes, 1993). Transmitters or other forms of activity-dependent stimuli that alter the expression and/or distribution of these types of surface molecules may thus alter the normal pattern of cellcell interactions critical for cellular plasticity.

Why do SN varicosities form at apCAM "hot spots" on L7 during either the initial formation of connections or following 5-HT applications? One possibility is that apCAM hot spots on the motor axon may retard or stop the extension of $\mathrm{SN}$ growth 
Figure 5. Transmitters alter apCAM levels prior to changes in $\mathrm{SN}$ structure. $A$, FMRFamide evokes a decline in apCAM expression at existing SN varicosities prior to detectable changes in SN structure. $A l$ and $A 3$, Rhodamine fluorescent views of SN neurites and varicosities regenerated by day 5 , prior $(A l)$ or after $(3 \mathrm{hr})$ the onset of treatments with FMRFamide (A3). Arrows point to two varicosities. $A 2$ and $A 4$, Pseudocolor representation of the FITC-anti-apCAM staining in the same area, before $(A 2)$ and after $(A 4)$ treatments with FMRFamide. Note that the FITC-anti-apCAM staining in the same area has undergone a substantial decrease, especially at varicosities indicated by the arrows where intensities have declined from yellow-red to bluegreen. $B, 5$-HT begins to evoke an increase in apCAM levels at SN varicosities immediately after treatment. $B I$ and $B 3$, Rhodamine fluorescent views of $\mathrm{SN}$ neurites and varicosities before $(B 1)$ and immediately $(3 \mathrm{hr})$ after (B3) the onset of 5-HT treatments. Arrows point to two $\mathrm{SN}$ varicosities. $B 2$ and B4, Pseudocolor representation of FITC-anti-apCAM staining in the same area before $(B 2)$ and $3 \mathrm{hr}$ after (B4) the onset of 5-HT treatments. Note that immediately following 5-HT treatment, there is no obvious change in SN structure (compare $B I$ and $B 3$ ). The overall apCAM level does not change (compare $B 2$ and $B 4$ ), but apCAM levels begin to increase (green-yellow to yellow) in areas with $\mathrm{SN}$ varicosities that are indicated by the arrows. Scale bar, $30 \mu \mathrm{m}$.
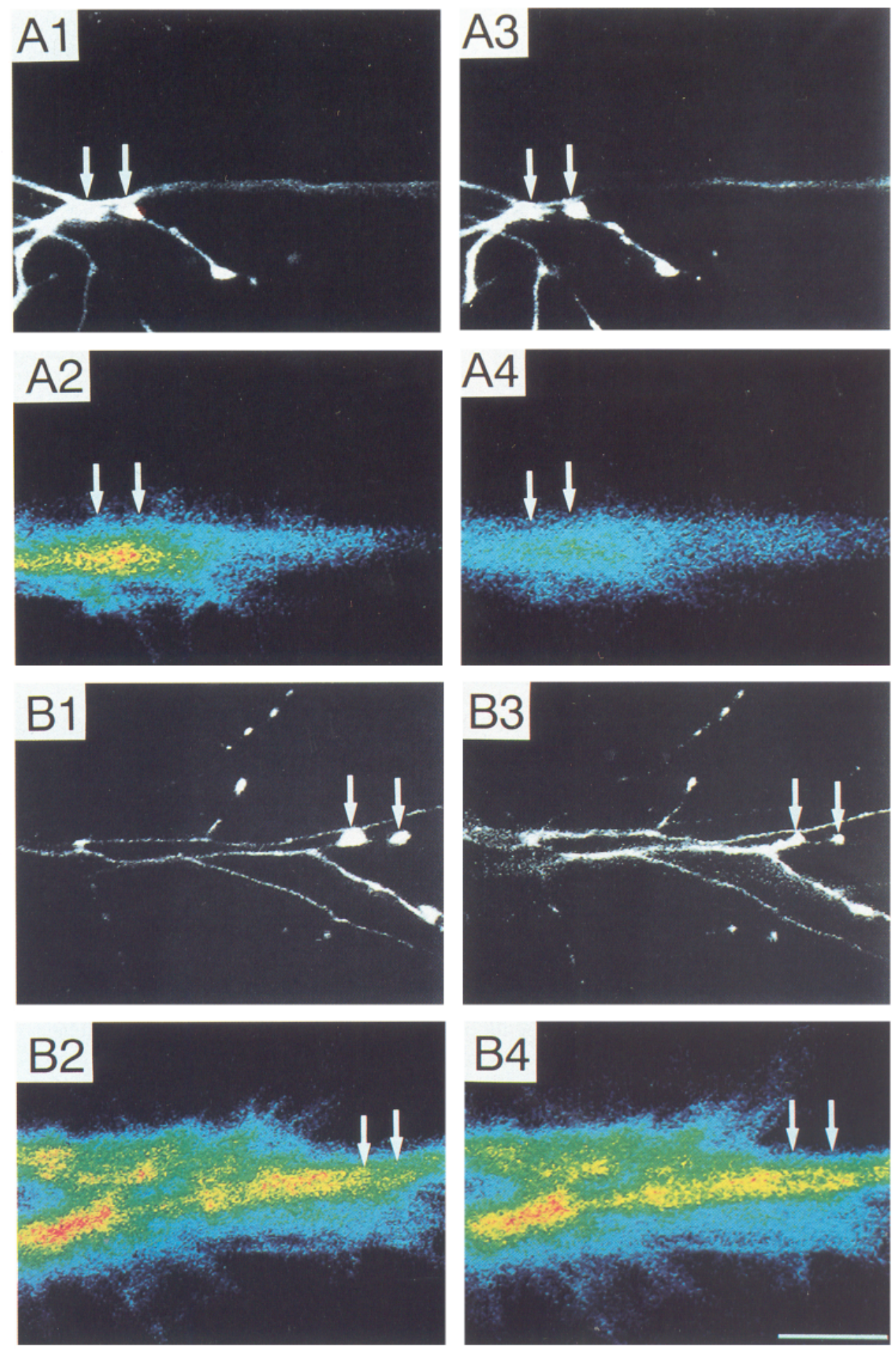

cones. The stopping of growth cone extension may be an important preliminary step in the formation of synaptic connections such as the neuromuscular junction (Landmesser, 1980; Keshishian et al., 1993) or mossy fiber terminals in the cerebellum (Baird et al., 1992a,b). The hot spots may influence the cycle of adhesion and defasciculation by growth cones as they extend on a target substrate. Rate of extension for some neurites maintained in vitro show an inverse U-shape relationship with NCAM concentration (Doherty et al., 1990b), while regulating the levels of polysialic acid modification of NCAM can also govern growth rate and branching (Doherty et al., 1990a; Land- messer et al., 1990; Tang et al., 1992). The differences in the level of ligand-receptor binding of apCAM on the presynaptic versus postsynaptic surfaces could affect neuritic growth and synapse formation by differentially regulating the level of activation of various intracellular second-messenger cascades that could affect the local organization of the cytoskeleton within the growth cone, presynaptic varicosity, or postsynaptic cell (Kater and Mills, 1991; Atashi et al., 1992; Doherty and Walsh, 1992; Lohof et al., 1992; Funte and Haydon, 1993; Lin and Forscher, 1993; Wu and Goldberg, 1993).

One of the consequences of the actions of 5-HT on apCAM 


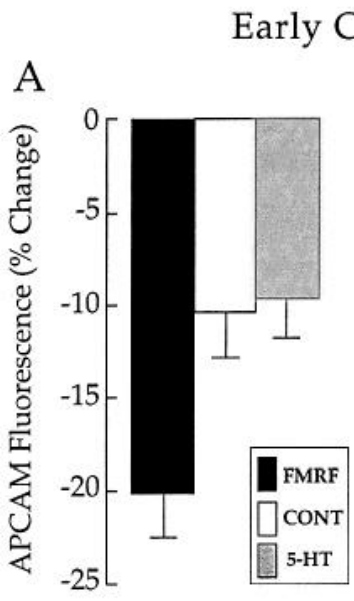

hanges in APCAM

B

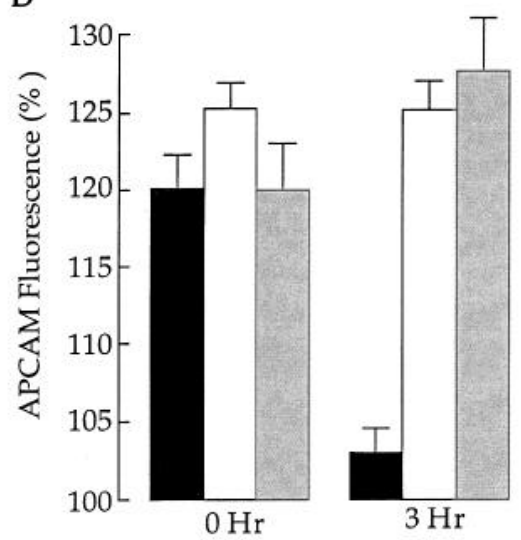

Figure 6. Summary of the early changes in apCAM expression $3 \mathrm{hr}$ after start of transmitter treatments. $A$, Overall apCAM levels along the motor axon containing SN neurites and varicosities is reduced significantly by FMRFamide. The height of each bar represents the mean \pm SEM of the percent change in apCAM fluorescence following different treatments. An ANOVA indicated a significant effect of treatment $[F(2,27)=6.499, p<0.01]$. Multicomparison $t$ tests (Dunnett's) indicated that the change with 5-HT was not different than control while FMRFamide decreased significantly apCAM levels $(t=3.223, p<$ 0.01). $B$, FMRFamide alters apCAM levels at $\mathrm{SN}$ varicosities. The height of each bar is the mean \pm SEM in the relative apCAM fluorescence intensity at preexisting $\mathrm{SN}$ varicosities before $(0 \mathrm{hr})$ and after $(3$ hr) the onset of the treatments. A two-factor ANOVA indicated a significant effect of treatment $[F(2,27)=32.803, p<0.001]$. An ANOVA at each time point indicated that there was no significant difference between the groups on $0 \mathrm{hr}[F(2,27)=1.644, p>0.2]$ while there was a significant effect of treatment at $3 \mathrm{hr}[F(2,27)=30.497, p<0.001]$. Multicomparison $t$ tests (Dunnett's) indicated that the increase with 5-HT was not significantly different than control at this early time point, but treatment with FMRFamide was significantly different than control $(t=6.39, p<0.01)$

levels is a further enrichment of apCAM at existing sites of $\mathrm{SN}$ varicosities that begins by $1 \mathrm{hr}$ after treatment and is changed significantly by $24 \mathrm{hr}$. 5-HT also evokes a rapid downregulation of apCAM synthesis and expression on the sensory neurites via an increase in endocytosis that is mediated by an increase in the activity of cAMP dependent PKA (Barzilai et al., 1989; Bailey et al., 1992a; Mayford et al., 1992; Hu et al., 1993; Peter et al., 1994; Wu and Schacher, 1994). The selective change in apCAM synthesis and expression on the surfaces of the SN neurites (Barzilai et al., 1989; Mayford et al., 1992; Peter et al., 1994) coupled with the enrichment of apCAM at preexisting varicosities and the presence of enriched apCAM zones on L7 would together lead to a decrease in the interaction between SN neurites with each other and increase further the "attractive" nature of the motor cell substrate facilitating the formation of new synaptic connections (Zhu et al., 1994). An important feature of that substrate for synapse formation can be abolished by incubating the cells in anti-apCAM mAb. Exposure to the mAb blocks the long-term structural and functional changes normally evoked by 5-HT, but does not interfere with the function of the preexisting connections in terms of the efficacy of transmission or its ability to undergo short-term modulation. The nonuniform distribution and high levels of apCAM expression on the proximal motor axon is one important factor affecting SN growth and synapse formation (Zhu et al., 1994) and may represent one of the critical factors underlying the observations that the structural changes in the SNs evoked by 5-HT or FMRFamide are dependent on the presence of the target motor cell (Glanzman et al., 1990; Schacher and Montarolo, 1991).

Although it is not possible at this time to quantify the contribution provided by each cell to levels of apCAM expressed at $\mathrm{SN}$ varicosities contacting the proximal motor axons, by extrapolating from the levels expressed by the isolated cells (Zhu et al., 1994), or from the levels expressed by each cell in those regions of the cocultures where it is possible to identify each cell (see Figs. 1, 3, 5), it is likely that most of the fluorescent signal is generated by apCAM on the surface of the motor cell. Thus, several lines of evidence suggest that FMRFamide-evoked changes in the distribution of apCAM on L7 at synaptic sites may contribute to the elimination of the presynaptic SN varicosities. First, most of the downregulation of apCAM from the surface of L7 occurs rapidly, by $1 \mathrm{hr}$ after the end of transmitter application (see also Peter et al., 1994; Wu and Schacher, 1994), and prior to changes in the structure of the cells. These changes parallel those observed for synapse elimination at the neuromuscular junction where removal of transmitter receptors from the surface of the muscle precedes retraction of the overlying nerve terminal (Rich and Lichtman, 1989; Balice-Gordan and Lichtman, 1993). Second, blocking the downregulation of apCAM in $\mathrm{L} 7$ by blocking FMRFamide activation of PKA also blocked the functional and structural changes evoked by FMRFamide (Wu and Schacher, 1994). Third, when summed together, the various isoforms of apCAM represent the major membrane protein in the nervous system of Aplysia (Keller and Schacher, 1990). The formation of SN varicosities at apCAM "hot spots" on L7 and the enrichment of apCAM at stable synaptic sites support the idea that these molecules are likely to play an important role in synapse maintenance. Fourth, blocking apCAM on $\mathrm{L} 7$ with $\mathrm{mAb}$ prior to sensory innervation significantly interferes with synapse formation (Zhu et al., 1994). Although we cannot rule out the involvement of other surface molecules that may be present or enriched at synaptic sites and colocalize with apCAM, it is likely that the changes in apCAM levels on L7 play a role in synapse elimination by FMRFamide.

The downregulation of apCAM from the SN neurites by 5-HT is not evident in SN-L7 cultures, since most of the apCAM signal is generated by the motor cell. The overall decline in apCAM signal with 5-HT was comparable to the change observed following control treatment. However, preexisting varicosities showed an increase in apCAM levels relative to the overall expression in the 5-HT group, indicating a slower rate of turnover at the varicosities. The apparent difference at the varicosities may arise for several reasons. First, apCAM on the surface of the presynaptic SN varicosities may be spared from downregulation by 5 -HT because it is selectively recycled back to the surface, whereas apCAM endocytosed at extrasynaptic regions are, for the most part, degraded (Bailey et al., 1992). Alternatively, 5-HT may initiate endocytosis of apCAM selectively from extrasynaptic sites while sparing sensory varicosities. This could arise by restricting specific isoforms of apCAM at the presynaptic SN varicosities (Faivre-Sarrailh et al., 1992) that are not endocytosed with 5-HT treatment. Third, apCAM levels at synaptic sites are retained after downregulation by 5-HT via the redistribution of remaining molecules on both the pre- and postsynaptic side of the varicosity (Singer, 1992). Regardless of these possibilities, which may be resolved with electron microscopic examination, the action of 5-HT on apCAM distribution may not only allow for the formation of new syn- 
apses, but also increase the stability of preexisting connections so that they are not disrupted as 5-HT increases endocytic activity in the sensory cell (Bailey et al., 1992; Hu et al., 1993).

In summary, our results suggest the possibility that the continuous expression of cell surface molecules throughout adult life that initially had important roles during the early stages of development in controlling aspects of cell migration, axonogenesis, and synaptogenesis, may continue to play a role in some of those same cellular processes that are associated with regencration or activity-dependent bchavioral plasticity.

\section{References}

Abrams TW, Castellucci VF, Camardo JS, Kandel ER, Lloyd PE (1984) Two endogenous neuropeptides modulate the gill and siphon withdrawal reflex in Aplysia by presynaptic facilitation involving cAMPdependent closure of a serotonin-sensitive potassium channel. Proc Natl Acad Sci USA 81:7956-7960.

Alvarez-Buylla A, Kirn JR, Nottebohm F (1990) Birth of projection neurons in adult avian brain may be related to perceptual or motor learning. Science 249:1444-1446.

Atashi JR, Klinz SG, Ingraham CA, Matten WT, Schachner M, Maness PF (1992) Neural cell adhesion molecules modulate tyrosine phosphorylation of tubulin in nerve growth cone membranes. Neuron $8: 831-842$.

Bailey CH, Chen M (1983) Morphological basis of long-term habituation and sensitization. Science 220:91-93.

Bailey CH, Chen M (1988) Long-term memory in Aplysia modulates the total number of varicosities of single identified sensory neurons. Proc Natl Acad Sci USA 85:2373-2377.

Bailey CH, Kandel ER (1993) Structural changes accompanying memory storage. Annu Rev Physiol 55:397-426.

Bailey CH, Chen M, Keller F, Kandel ER (1992a) Serotonin-mediated endocytosis of apCAM: an early step of learning-related synaptic growth in Aplysia. Science 256:645-648.

Bailey CH, Montarolo PG, Chen M, Kandel ER, Schacher S (1992b) Inhibitors of protein and RNA synthesis block the structural changes that accompany long-term heterosynaptic plasticity in the sensory neurons of Aplysia. Neuron 9:749-758.

Baird DH, Baptista CA, Wang LC, Mason CA (1992a) Specificity of a target-derived stop signal for afferent axon growth. J Neurobiol 23: 579-591.

Baird DH, Hatten ME, Mason CA (1992b) Cerebellar target neurons provide a stop signal for afferent neurite extension in vitro. J Neurosci 12:619-634.

Balice-Gordan RJ, Lichtman JW (1993) In vivo observations of preand postsynaptic changes during the transition from multiple to single innervation at developing neuromuscular junctions. J Neurosei 13: 834-855.

Bank M, Schacher S (1992) Segregation of presynaptic inputs on an identified target neuron in vitro: structural remodeling visualized over time. J Neurosci 12:2960-2972.

Barzilai A, Kennedy TE, Sweatt JD, Kandel ER (1989) 5-HT modulates protein synthesis and the expression of specific proteins during long-term facilitation in Aplysia sensory neurons. Neuron 2:15771586 .

Belardetti F, Kandel FR, Siegelhaum SA (1987) Neuronal inhibition by the peptide FMRFamide involves opening of S-potassium channels. Nature 325:153-156.

Brunelli M, Castellucci VF, Kandel ER (1976) Synaptic facilitation and behavioral sensitization in Aplysia: possible role of serotonin and cyclic AMP. Science 194:1178-1181.

Castellucci VF, Carew TJ, Kandel ER (1978) Cellular analysis of longterm habituation of the gill-withdrawal reflex in Aplysia. Science 202: $1306-1308$

Chow I (1990) Cell-cell interaction during synaptogenesis. J Physiol (Paris) 84:121-127.

Clark GA, Kandel ER (1993) Induction of long-term facilitation in Aplysia sensory neurons by local application of serotonin to remote synapses. Proc Natl Acad Sci USA 90:11411-11415.

Cremer H, Lange R, Christoph A, Plomann M, Vopper G, Roes J, Brown R, Baldwin S, Kraemer P, Scheff S, Barthels D, Rajewsky K, Wille W (1994) Inactivation of the N-CAM gene in mice results in size reduction of the olfactory bulb and deficits in spatial learning. Nature 367:455-459

Dale N, Schacher S, Kandel ER (1988) Long-term facilitation in Aplysia involves increase in transmitter release. Science 239:282-285.

Daniloff JK, Levi G, Grumet M, Rieger F, Edelman GM (1986) Altered expression of neuronal cell adhesion molecules induced by nerve injury and repair. J Cell Biol 103:929-945.

Doherty P, Walsh FS (1992) Cell adhesion molecules, second messengers and axonal growth. Curr Opin Neurobiol 2:595-601.

Doherty P, Cohen J, Walsh FS (1990a) Neurite outgrowth in response to transfected NCAM changes during development and is modulated by polysialic acid. Neuron 5:209-219.

Doherty P, Fruns M, Seaton P, Dickson G, Barton CH, Sears TA, Walsh FS (1990b) A threshold effect of the major isoforms of NCAM on neurite outgrowth. Nature 343:464-466.

Edelman GM (1983) Cell adhesion molecules. Science 219:450-457.

Edelman GM, Cunningham BA (1991) Place-dependent cell adhesion, process retraction, and spatial signalling in neural morphogenesis. Cold Spring Harbor Symp Quant Biol 55:303-318.

Emptage NJ, Carew TJ (1993) Long-term synaptic facilitation in the absence of short-term facilitation in Aplysia neurons. Science 262 $253-256$.

Faivre-Sarrailh C, Gennarini G, Goridis C, Rougon G (1992) F3/F11 cell surface molecule expression in the developing mouse cerebellum is polarized at synaptic sites and with granule cells. J Neurosci 12 257-267

Frost WN, Castellucci VF, Hawkins RD, Kandel ER (1985) The monosynaptic connections made by the sensory neurons of the gill- and siphon-withdrawal reflex participate in the storage of long-term memory for sensitization. Proc Natl Acad Sci USA 82:8266-8270.

Funte LR, Haydon PG (1993) Synaptic target contact enhances presynaptic calcium influx by activating cAMP-dependent protein kinase during synaptogenesis. Neuron 10:1069-1078.

Gilbert CD (1993) Rapid dynamic changes in adult cerebral cortex. Curr Opin Neurobiol 3:100-103.

Glanzman DL, Kandel ER, Schacher S (1989a) Identified target motor neuron regulates neurite outgrowth and synapse formation of Aplysia sensory neurons in vitro. Neuron 3:441-450.

Glanzman DL, Mackey SL, Hawkins RD, Dyke AM, Lloyd PE, Kandel ER (1989b) Depletion of serotonin in the nervous system of Aplysia reduces the behavioral enhancement of gill withdrawal as well as the heterosynaptic facilitation produced by tail shock. J Neurosci 9:4200_ 4213.

Glanzman DL, Kandel ER, Schacher S (1990) Target-dependent structural changes accompanying long-term synaptic facilitation in Aplysia neurons. Science 249:799-802.

Glanzman DL, Kandel ER, Schacher S (1991) Target-dependent morphological segregation of Aplysia sensory outgrowth in vitro. Neuron 7:903-913.

Hall ZW, Sanes JR (1993) Synaptic structure and development: the neuromuscular junction. Cell 72/Neuron 10:99-122.

Hawver DB, Schacher S (1993) Selective fasciculation as a mechanism for the formation of specific chemical connections between Aplysia in vitro. J Neurobiol 24:368-383.

Hortsch M, Goodman CS (1991) Cell and substrate adhesion molecules in Drosophila. Annu Rev Cell Biol 7:505-557.

Hu Y, Barzilai A, Chen M, Bailey CH, Kandel ER (1993) 5-HT and cAMP induce the formation of coated pits and vesicles and increase the expression of clathrin light chain in sensory neurons of Aplysia. Neuron 10:921-929.

Keller F, Schacher S (1990) Neuron-specific membrane glycoproteins promoting neurite fasciculation in Aplysia californica. $\mathbf{J}$ Cell Biol 111:2637-2650

Key B, Akeson RA (1991) Delineation of olfactory pathways in the frog nervous system by unique glycoconjugates and NCAM glycoforms. Neuron 6:381-396.

Kishishian H, Chiba A, Chang TN, Halson MS, Harkins EW, Jarecki J, Wang L, Anderson M, Cash S, Halpern ME, Johanson J (1993) Cellular mechanisms governing synaptic development in Drosophila melanogaster. J Neurobiol 24:757-787.

Landmesser LT (1980) The generation of neuromuscular specificity. Annu Rev Neurosci 3:279-302.

Landmesser LT, Dahm L, Tang J, Rutishauser U (1990) Polysialic acid as a regulator of intramuscular nerve branching during embryonic development. Neuron 4:655-667. 
Lin CH, Forscher P (1993) Cytoskeletal remodeling during growth cone-target interactions. J Cell Biol 121:1369-1383.

Lohof AM, Quillan M, Day Y, Poo M-M (1992) Asymmetric modulation of cytosolic cAMP activity induces growth cone turning. $J$ Neurosci 12:1253-1261.

Mackey SL, Glanzman DL, Small SA, Dyke AM, Kandel, ER, Hawkins RD (1987) Tail shock produces inhibition as well as sensitization of the siphon withdrawal reflex of Aplysia: possible behavioral role for presynaptic inhibition mediated by the peptide FMRFamide. Proc Natl Acad Sci USA 84:8730-8734.

Mayford M, Barzilai A, Keller F, Schacher S, Kandel ER (1992) Modulation of an NCAM-related adhesion molecule with long-term synaptic plasticity in Aplysia. Science 256:638-644.

McMahan UJ, Horton SE, Werle MJ, Honig LS, Kroger S, Ruegg MA Escher G (1992) Agrin isoforms and their role in synaptogenesis. Curr Opin Cell Biol 4:869-874.

Merzenich MM, Sameshima K (1993) Cortical plasticity and memory. Curr Opin Neurobiol 3:187-196.

Miragall F, Kadmon G, Husmann M, Schachner M (1988) Expression of cell adhesion molecules in the olfactory system of the adult mouse: presence of the embryonic form of NCAM. Dev Biol 129:516-531.

Montarolo PG, Goelet P, Castellucci VF, Morgan J, Kandel ER, Schacher S (1986) A critical time window for macromolecular synthesis in long-term heterosynaptic facilitation in Aplysia. Science 234:12491254.

Montarolo PG, Kandel ER, Schacher S (1988) Long-term heterosynaptic inhibition in Aplysia. Nature 333:171-174.

Nottebohm F (1991) Reassessing the mechanisms and origins of vocal learning in birds. Trends Neurosci 14:206-211.

Persohn E Schachner, M (1990) Immunohistological localization of the neural cell adhesion molecules L1 and NCAM in the developing hippocampus of the mouse. J Neurocytol 19:807 819.

Peter N, Aronoff B, Wu F, Schacher S (1994) Decrease in growth coneneurite fasciculation by sensory or motor cells in vitro accompanies downregulation of Aplysia cell adhesion molecule by neurotransmitters. J Neurosci 14:1413-1421.

Pieroni JP, Byrne JH (1992) Differential effects of serotonin, FMRFamide, and small cardioactive peptide on multiple, distributed processes modulating sensorimotor synaptic transmission in Aplysia. J Neurosci 12:2633-2647

Pollerberg EG, Sadoul R, Goridis C, Schachner M (1985) Selective expression of the $180-\mathrm{kD}$ component of the neural cell adhesion molecule NCAM during development. J Cell Biol 101:1921-1929.

Rayport SG, Schacher S (1986) Synaptic plasticity in vitro: cell culture of identified Aplysia neurons mediating short-term habituation and sensitization. J Neurosci 6:759-763.

Rich MM, Lichtman JW (1989) In vivo visualization of pre- and post- synaptic changes during synapse elimination in reinnervated mouse muscle. J Neurosci 9:1781-1805.

Rutishauser U, Acheson A, Hall A, Mann D, Sunshine J (1988) The neural cell adhesion molecule (NCAM) as a regulator of cell-cell interactions. Science 240:53-57.

Schacher S (1985) Differential synapse formation and neuritic outgrowth at two branches of the metacerebral cell of Aplysia in dissociated cell culture. J Neurosci 5:2028-2034.

Schacher S, Proshansky E (1983) Neurite regeneration by Aplysia neurons in dissociated cell culture: modulation by Aplysia hemolymph and the presence of the initial axon segment. J Neurosci 3:24032413.

Schacher S, Montarolo PG (1991) Target-dependent structural changes in sensory neurons of Aplysia accompany long-term heterosynaptic inhibition. Neuron 6:679-690.

Schacher S, Montarolo PG, Kandel ER (1990) Selective short- and long-term effects of serotonin, small cardioactive peptide, and tetanic stimulation on sensorimotor synapses of Aplysia in culture. J Neurosci 10:3286-3294.

Schacher S, Glanzman DL, Barzilai A, Dash P, Grant SGN, Keller F, Mayford M, Kandel ER (1991) Long-term facilitation in Aplysia: persistent phosphorylation and structural changes. Cold Spring Harbor Symp Quant Biol 55:187-202.

Scholey AB, Rose SPR, Zamani MR, Block E, Schachner M (1993) A role for the neural cell adhesion molecule in a late, consolidation phase of glycoprotein synthesis six hours following passive avoidance training of the young chick. Neuroscience 55:499-509.

Seki T, Arai Y (1993) Highly polysialated neural cell adhesion molecule (NCAM-H) bis expressed by newly generated granule cells in the dentate gyrus of the adult rat. J Neurosci 13:2351-2358.

Singer SJ (1992) Intracellular communication and cell-cell adhesion. Science 255:1671-1677.

Small SA, Cohen TE, Kandel ER, Hawkins RD (1992) Identified FMRFamide-immunoreactive neuron LPL16 in the left pleural ganglion of Aplysia produces presynaptic inhibition of siphon sensory neurons. J Neurosci 12:1616-1627.

Tang J, Landmesser L, Rutishauser U (1992) Polysialic acid influences specific pathfinding by avian motoneurons. Neuron 8:1031-1044.

Tomasiewicz H, Ono K, Yee D, Thompson C, Goridis C, Rutishauser U, Magnuson T (1993) Genetic deletion of a neural cell adhesion molecule variant (NCAM-180) produces distinct defects in the central nervous system. Neuron 11:1163-1174.

Wu D-Y, Goldberg DJ (1993) Rapid tyrosine phosphorylation at the tips of growth cone filopodia. J Cell Biol 123:653-664.

Wu F, Schacher S (1994) Pre- and postsynaptic changes mediated by two second messengers contribute to the expression of Aplysia longterm heterosynaptic inhibition. Neuron 12:407-421.

Zhu H, Wu F, Schacher S (1994) Aplysia cell adhesion molecules and serotonin regulate sensory cell-motor cell interactions during carly stages of synapse formation in vitro. J Neurosci 14:6886-6900. 